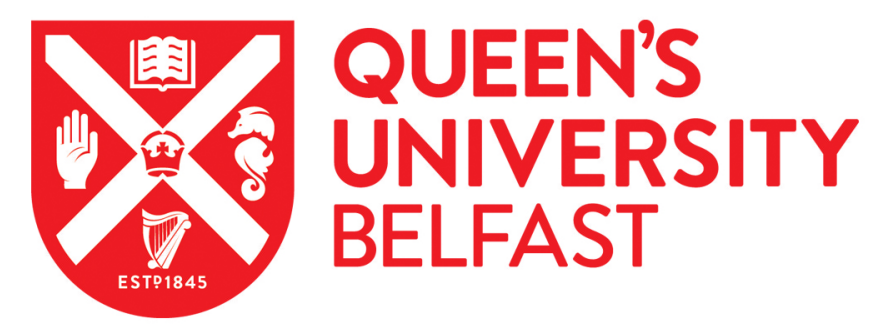

\title{
Adaptive calibration of a three-microphone system for acoustic waveguide characterization under time-varying conditions
}

Walstijn, M. V., \& Sanctis, G. D. (2014). Adaptive calibration of a three-microphone system for acoustic waveguide characterization under time-varying conditions. Journal of the Acoustical Society of America, 135(2), 917-927. https://doi.org/10.1121/1.4861250

Published in:

Journal of the Acoustical Society of America

Document Version:

Publisher's PDF, also known as Version of record

Queen's University Belfast - Research Portal:

Link to publication record in Queen's University Belfast Research Portal

\begin{abstract}
Publisher rights
Copyright (2014) Acoustical Society of America. This article may be downloaded for personal use only. Any other use requires prior permission of the author and the Acoustical Society of America. The following article appeared in Walstijn, M. V., \& Sanctis, G. D. (2014) Adaptive calibration of a three-microphone system for acoustic waveguide characterization under time-varying conditions. Journal of the Acoustical Society of America, 135(2), 917-927 and may be found at http://dx.doi.org/10.1121/1.4861250
\end{abstract}

\section{General rights}

Copyright for the publications made accessible via the Queen's University Belfast Research Portal is retained by the author(s) and / or other copyright owners and it is a condition of accessing these publications that users recognise and abide by the legal requirements associated with these rights.

Take down policy

The Research Portal is Queen's institutional repository that provides access to Queen's research output. Every effort has been made to ensure that content in the Research Portal does not infringe any person's rights, or applicable UK laws. If you discover content in the Research Portal that you believe breaches copyright or violates any law, please contact openaccess@qub.ac.uk. 
Copyright (2014) Acoustical Society of America. This article may be downloaded for personal use only. Any other use requires prior permission of the author and the Acoustical Society of America.

The following article appeared in (citation of published article) and may be found at

http://scitation.aip.org/content/asa/journal/jasa/135/2/10.1121/1.4861250. 


\title{
Adaptive calibration of a three-microphone system for acoustic waveguide characterization under time-varying conditions
}

\author{
Maarten van Walstijn ${ }^{\text {a) }}$ and Giovanni de Sanctis \\ School of Electronics, Electrical Engineering, and Computer Science, Queen's University Belfast, \\ Belfast, BT7 INN, United Kingdom
}

(Received 3 October 2013; revised 6 December 2013; accepted 12 December 2013)

\begin{abstract}
The pressure and velocity field in a one-dimensional acoustic waveguide can be sensed in a non-intrusive manner using spatially distributed microphones. Experimental characterization with sensor arrangements of this type has many applications in measurement and control. This paper presents a method for measuring the acoustic variables in a duct under fluctuating propagation conditions with specific focus on in-system calibration and tracking of the system parameters of a three-microphone measurement configuration. The tractability of the non-linear optimization problem that results from taking a parametric approach is investigated alongside the influence of extraneous measurement noise on the parameter estimates. The validity and accuracy of the method are experimentally assessed in terms of the ability of the calibrated system to separate the propagating waves under controlled conditions. The tracking performance is tested through measurements with a time-varying mean flow, including an experiment conducted under propagation conditions similar to those in a wind instrument during playing. (C) 2014 Acoustical Society of America. [http://dx.doi.org/10.1121/1.4861250]
\end{abstract}

PACS number(s): 43.75.Yy, 43.58.Vb, 43.58.Bh [JW]

Pages: $917-927$

\section{INTRODUCTION}

Experimental characterization of one-dimensional acoustic waveguides has seen much interest over the years, finding application in several fields, including noise control, nondestructive testing, absorption measurement, and musical acoustics. Various measurement techniques and setups have been proposed, differing mainly in the manner of excitation and the number of sensors. The target response usually takes the form of an acoustic impedance ${ }^{1,2}$ or reflection function ${ }^{3,4}$ at a chosen reference section. Alternatively the problem can be framed as the determination of a transfer matrix., Several of such methods have been designed to take measurements in the presence of a steady mean flow. ${ }^{7-11}$ In most cases, the measurements are "non-intrusive" in the sense that wall flush mounted pressure sensors of near-zero input admittance are employed, meaning that minimal interference with the acoustic field can be assumed.

The majority of techniques involve a pre-calibration of the measurement system. This is particularly useful when the object under study has a highly resonant nature, such as a musical wind instrument air column, leading to specific requirements on the frequency resolution and dynamic range. $^{2,12}$ The literature provides several comprehensive overviews of calibration methods for the possible experimental setups. ${ }^{4,12-14}$ Generally the calibration procedures rely on having constant conditions during the experiment and as such are not suited to characterizing the system under any fluctuations in temperature, humidity or mean flow. The ability to measure in such circumstances is, for example, of interest when the aim is to seek information about the

\footnotetext{
a) Author to whom correspondence should be addressed. Electronic mail: m.vanwalstijn@qub.ac.uk
}

acoustic functioning and control of a wind instrument, including the interaction with the reed or lip. ${ }^{15-17}$ Another application that requires a more adaptive approach to calibration is the measurement of a transient mean flow in a duct using pressure sensors.

The aim of the present study is to develop a nonintrusive method for measuring the acoustic variables in a duct under time-varying propagation conditions, which, under the plane-wave assumption, translates to the problem of separating the traveling waves. In a sense, all onedimensional (1-D) duct measurement techniques can be considered as wave separation methods because once the impedance and the pressure are known at a specific section, the particle velocity can be determined, from which the forward and backward propagating waves are directly obtained. Nevertheless relatively few studies have been aimed at directly addressing the wave separation problem. Among these, a closely related work is that by Guérard and Boutillon, ${ }^{18,19}$ who developed a multiple-microphone technique in which the normalized particle velocity is estimated as a finite-difference approximation of the pressure gradient. Because the discrete derivative is simply a weighted sum of the microphone outputs, a real-time implementation is straightforward with analog electronics, making the method particularly suited to active control. The wave separation problem can also be approached from a stochastic perspective, which has the advantage of giving a direct handle on the effect of measurement noise on the performance. This approach has recently been investigated through theory and simulations by Nauclér and Söderström ${ }^{20}$ within the constraints typical of control applications. Another recent study of direct relevance is the investigation into nonlinear wave propagation by Rendón et $a .^{21}$ in which pulse waves in a trombone are successfully separated using a technique based 
on the classic two-microphone method. ${ }^{7}$ However, none of these methods adapt to the conditions, and in all cases, the estimation accuracy is compromised somewhat by neglecting the propagation losses. Kemp et al. ${ }^{22}$ perform wave separation using a time-domain method in which the propagation losses are taken into account, with recent application to a trumpet under playing conditions. ${ }^{23}$ However, the calibration relies again on having constant conditions, which complicates any modification toward adaptive calibration and limits the technique to studying brief notes.

The novelty of the challenge here is that the fluctuating conditions themselves can generally not be repeated, thus any evolving aspects must be captured instantly from a single acquisition. In addition, the oscillations in the duct may not be under any kind of precise control. This immediately rules out a full pre-calibration of the complete measurement system via prior measurements and also heavily limits the scope for reducing noise effects via averaging. The present authors propose to address the problem by making use of the considerable amount of a priori information about the physical behavior of the system. That is, the measurement system is modeled and characterized across a broad frequency range by a few physical parameters that are then to be estimated. This parametric strategy transforms the calibration question into a nonlinear optimization problem in which one can distinguish between condition-independent parameters, which can be pre-calibrated, and condition-dependent parameters, which need to be tracked over time.

The development of the proposed method centers around the concept of two-microphone wave separation, which represents an appropriately general target problem. In addition, it provides the basis for the formulation of a suitable optimization cost function as well as for the definition of a new validation metric. The calibration is based on the principle of minimizing the difference between two separate estimates of the forward pressure wave as obtained from two microphone pairs within a three-microphone configuration.

The paper is organized as follows. Section II reviews the theory underlying the wave separation problem and presents a three-microphone measurement arrangement that affords calibration. In Sec. III, the estimation of the parameters via optimization is explained. Section IV then discusses experiments designed to assess the performance of the proposed method under controlled conditions. Finally in Sec. V, the tracking performance is tested through measurements with mean flow, including an experiment in which the air flow is supplied by human breath, thus sensing the acoustic variables under fluctuations in temperature, humidity, and mean flow.

\section{WAVE SEPARATION}

In the following, wave propagation is assumed to be linear, and frequency-domain variables and system transfer functions are written with upper-case letters, omitting the dependence on frequency in the notation. For example, $P(x)$ denotes the Fourier transform $P(x, \omega)$ of the time domain pressure signal $p(x, t)$.

\section{A. Two-microphone wave separation}

Consider an acoustic duct with two microphones $\left(\mathcal{M}_{a}\right.$ and $\mathcal{M}_{b}$ ) embedded in its wall (see Fig. 1). From the planewave assumption, the pressure field inside the duct is described by

$$
P(x)=\underbrace{A \exp (-\Gamma x)}_{P^{+}(x)}+\underbrace{B \exp (\Gamma x)}_{P^{-}(x)},
$$

where $x$ is the position along the duct, $\Gamma$ is the propagation constant, and $A$ and $B$ are the complex Fourier amplitudes of the traveling waves. From evaluating (1) at the microphone positions, and assuming ideal microphones with flat amplitude and zero phase response, the sensed pressures can be expressed in terms of the traveling waves at the position of $\mathcal{M}_{a}$ as

$$
\left[\begin{array}{l}
P_{a} \\
P_{b}
\end{array}\right]=\left[\begin{array}{cc}
1 & 1 \\
H & H^{-1}
\end{array}\right]\left[\begin{array}{c}
P_{a}^{+} \\
P_{a}^{-}
\end{array}\right],
$$

where $H=\exp (-\Gamma d)$ is a transfer function representing the propagation of a wave over distance $d=x_{b}-x_{a}$, and where $P_{a}$ denotes $P\left(x_{a}\right)$. By inverting (2), an estimate of the forward traveling wave can be obtained from the microphone signals as ${ }^{21,22,24}$

$$
\hat{P}_{a}^{+}=\frac{P_{a}-H P_{b}}{1-H^{2}} .
$$

The backward traveling pressure wave estimate can then be determined as $\hat{P}_{a}^{-}=P_{a}-\hat{P}_{a}^{+}$, and subsequently the pressure waves at any position $x$ can be obtained by multiplying with the appropriate transfer functions.

It is directly seen from (3) that the estimation error due to measurement noise or other factors becomes large whenever $H$ is equal to or near unity. As with any twomicrophone method, this occurs whenever an integer multiple of half the wavelength equals or is close to the inter-microphone distance, meaning that the system in (2) becomes ill-conditioned. ${ }^{7}$ The inter-microphone distance $d$ must therefore be chosen such that the system can produce accurate results within a target frequency range between the singular frequencies $f=0$ and $f=c /(2 d)$, where $c$ is the wave velocity. A nominal distance of $d=2 \mathrm{~cm}$ is used in this study, which affords a separation bandwidth of about $8 \mathrm{kHz}$.

\section{B. Waveguide parameters}

Equation (3) can be used to obtain an estimate of the forward wave, provided that $H$ and therefore the term $\Gamma d$ is

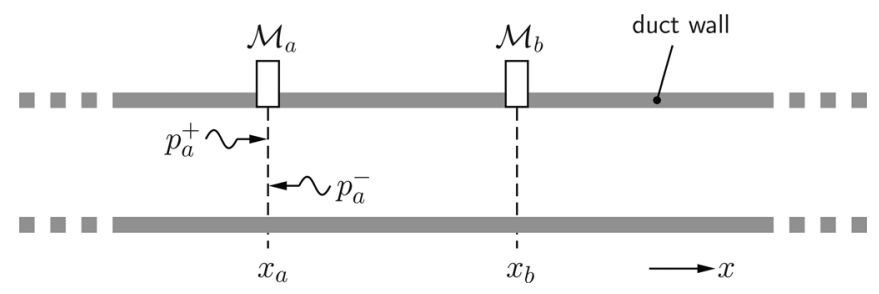

FIG. 1. An acoustic duct section with two wall-embedded microphones $\left(\mathcal{M}_{a}, \mathcal{M}_{b}\right)$ for separating the traveling waves. 
known. For pressure waves traveling in a cylindrical duct of sufficient diameter, such that the boundary layers occupy only a very small fraction of the duct's cross-sectional area, the propagation constant is ${ }^{25}$

$$
\Gamma=j\left(\frac{\omega}{c}\right)+(1+j) \alpha_{w},
$$

where $\alpha_{w}$ is the attenuation constant associated with viscous drag and heat conduction at the duct wall, which depends on the duct radius $a$ and the frequency $\omega$. Using Pierce's formula $^{25}$

$$
\alpha_{w}=\frac{1}{a} \sqrt{\frac{\eta \omega}{2 \rho c^{2}}}\left(1+\frac{\gamma-1}{v}\right),
$$

Eq. (4) can be rewritten as ${ }^{15,24}$

$$
\Gamma=\frac{1}{c}(j \omega+g \sqrt{j \omega}),
$$

where $g$ is a coefficient that embeds all the losses per unit length,

$$
g=\frac{1}{a} \sqrt{\frac{\eta}{\rho}}\left(1+\frac{\gamma-1}{v}\right),
$$

and where $\eta, \rho, \gamma$, and $v$, respectively, are the shear viscosity, the air mass density, the ratio of specific heats, and the square root of the Prandtl number. ${ }^{25,26}$ The transfer function may thus be written as a function of just two parameters $(\tau, g)$,

$$
H=\exp [-\tau(j \omega+g \sqrt{j \omega})]
$$

where $\tau=d / c$ represents the time it takes a lossless wave to travel over a distance $d$. Although more refined formulations of $\Gamma$ are possible, ${ }^{26}$ it can be verified experimentally ${ }^{27}$ that (4) is accurate for $|\Gamma a|<1$.

For estimation under fluctuating conditions, the presence of a small mean flow in the duct has to be considered, as is the case when air is blown into the bore of a wind instrument. This means that the propagation constant takes on a different form depending on the traveling direction ${ }^{9,11}$

$$
\Gamma^{+}=\frac{\Gamma}{1+M}, \quad \Gamma^{-}=\frac{\Gamma}{1-M}
$$

where $M$ is the mean flow Mach number, and where the superscripts "+" and "-" indicate the positive and the negative traveling direction, respectively. The expressions in (9) only hold for small Mach numbers $(M \ll 1)$, in which case the first-order Taylor approximation $(1+M)^{-1} \simeq 1-M$ can be employed in defining the transfer functions representing wave travel in either direction over a section distance $d=c \tau$,

$$
H^{ \pm}=\exp \left[-\frac{\tau}{1 \pm M}(j \omega+g \sqrt{j \omega})\right] \simeq H^{(1 \mp M)},
$$

where, on the right-hand side of (10), $H$ is the intermicrophone transfer function of section $m$ in the absence of mean flow as defined by (8).

\section{Three-microphone measurement configuration}

The waveguide parameters $(\tau, g, M)$ can be determined via calibration, but this requires additional information, which can be obtained by using more than two microphones. To limit the impact on the system under study, the number of microphones is kept to three. These are labeled $\mathcal{M}_{1}, \mathcal{M}_{0}$, and $\mathcal{M}_{2}$, and their relative positions along the duct can be seen in Figs. 2 and 6. The argument for restricting the number of microphones is particularly strong for wave separation in musical wind instruments, which generally have limited space regarding fitting microphones in the wall of any of its cylindrical bore sections.

The signal path for the pressure waves in the proposed three-microphone measurement configuration is depicted schematically in Fig. 2. Each block labeled $H_{m}^{ \pm}$models propagation of a pressure wave over an inter-microphone distance, and the corresponding wave travel times in the absence of a mean flow are denoted $\tau_{m}(m=1,2)$. To develop a practical measurement method, the following non-idealities are taken in account in comparison to the twomicrophone wave separation discussed in Sec. II A. First, the spacing between the microphones cannot be assumed to precisely equal the nominal value, thus $\tau_{1} \neq \tau_{2}$. Second, characteristics of the microphones and the acquisition system are accounted for by assigning (frequency-dependent) complex amplitudes $\left(G_{1}, G_{0}, G_{2}\right)$ to the acquisition channels. The number of additional system parameters to be estimated can be reduced by treating $\mathcal{M}_{0}$ as the reference microphone and defining two inter-channel complex amplitudes,

$$
\begin{aligned}
& F_{1}=\frac{G_{1}}{G_{0}}=a_{1} \exp \left(-j \omega \delta_{1} T\right), \\
& F_{2}=\frac{G_{2}}{G_{0}}=a_{2} \exp \left(-j \omega \delta_{2} T\right),
\end{aligned}
$$

where the frequency-independent constants $a_{1}, a_{2}$ and $\delta_{1}, \delta_{2}$, respectively, represent the inter-channel gains and delays, and $T$ is the sampling period. It is worth emphasizing that modeling the sensitivity differences between acquisition

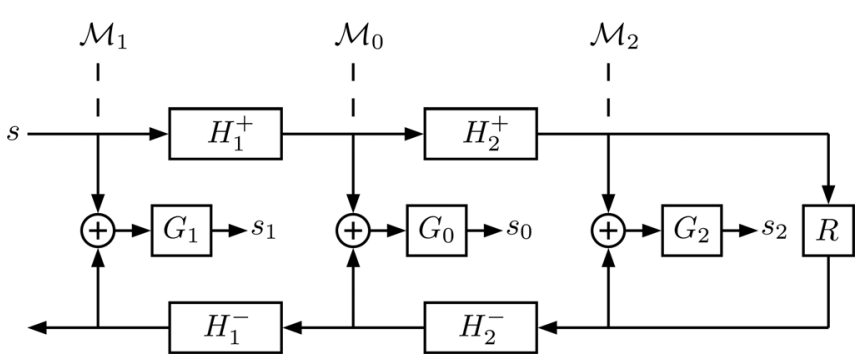

FIG. 2. Signal path for the pressure waves inside a duct section with three microphones. On the input side, $s$ represents the source signal injected into the duct section, first arriving at $\mathcal{M}_{1}$ as a forward-traveling wave. Each of the recorded microphone signals $s_{i}$ equals the local pressure filtered by the respective acquisition channel response $G_{i}(i=1,0,2) . R$ represents the reflection function as seen from $\mathcal{M}_{2}$. 
channels with simple constants does not imply that the microphones are assumed to have flat frequency responses; the only requirement is that the microphones have (nearly) identical characteristics, which tends to be sufficiently met when they are of the same type. Including the inter-channel delays is necessary whenever the employed digital acquisition system uses time-multiplexing (this the case for most low-cost multi-channel data acquisition systems), resulting into inter-channel sub-sample delays that need correcting to avoid estimation errors.

From the signal path in Fig. 2, and considering that $H^{+} H^{-}=H^{2}$, the generic two-microphone wave separation formula that replaces (3) after taking into account a mean flow and the non-idealities becomes

$$
\hat{P}_{a}^{+}=\frac{P_{a}-H^{(1+M)} P_{b}}{1-H^{2}}=\frac{S_{a} F_{a}^{-1}-H^{(1+M)} S_{b} F_{b}^{-1}}{G_{0}\left(1-H^{2}\right)} .
$$

\section{Frequency domain processing}

In principle it is possible to realize Eq. (13) in the time domain, which amounts to filtering of the discrete-time microphone signals $s_{a}[n]$ and $s_{b}[n]$, where $n$ is the time index. Similar linear filtering operations are required in the parameter estimation methods discussed in Sec. III. The common element in these signal processing operations is the modeling of propagation over an inter-microphone distance, which in the frequency domain is expressed as the multiplication with a transfer function of the form of (10). Even though accurate discrete-time formulations are possible, ${ }^{28,29}$ this invariably involves elaborate filter coefficient calculations for which closed-form expressions are generally not available. Hence time-domain processing would significantly complicate the calibration procedure as well as put limitations on it regarding the use of analytical gradient methods. For this reason, the proposed algorithms largely operate in the frequency domain, allowing direct evaluation of $H$ in its simple analytic frequency-domain form.

To obtain the Fourier transforms of the acquired microphone signals, the discrete Fourier transform (DFT) is used. Wave separation is performed on the resultant discrete spectra by first evaluating (8) and then applying (3), after which an inverse DFT is used to transform back to the time domain. Linear-phase post-process filtering is applied to remove any frequency components around and above the first singular frequency as well as any components at and near frequency zero. In the parameter estimation procedure, a smooth analysis window is applied before taking a DFT to reduce spectral leakage. Throughout the manuscript, presented simulation and measurement results are obtained using a $100 \mathrm{kHz}$ sampling frequency. The analysis window length is 8192 samples unless stated differently.

\section{E. Calibration and tracking}

The calibration problem to be addressed can now be stated as the estimation of eight system parameters, which, grouped into a vector, are

$$
\mathbf{v}=\left[\tau_{1}, r, g, a_{1}, a_{2}, \delta_{1}, \delta_{2}, M\right]
$$

where $r=\tau_{2} / \tau_{1}$ is the propagation time ratio. Before discussing how to best estimate $\mathbf{v}$, it is important to note that is not possible to estimate the entire parameter vector using a sole acquisition of microphone signals. This is because the effect that the inter-channel delays have on the system is equivalent and directly interchangeable to that of the mean flow, so the problem would be underdetermined. Therefore a distinction is made between tracking parameters and structural parameters. The latter category includes the propagation time ratio and the inter-channel gains and delays; these parameters can be considered as independent of mean flow, temperature, and humidity provided that sufficiently condition-proof sensors are employed. The parameters that, under time-varying conditions, are to be tracked are $\tau_{1}, g$, and $M$; the parameter $\tau_{2}$ can be determined subsequently as $\tau_{2}=r \tau_{1}$. For validation purposes, the methodology is first tested in Sec. IV for controlled, constant conditions without mean flow (thus setting $M=0$ ) in which case the estimation of seven parameters can be achieved from a single acquisition.

\section{PARAMETER ESTIMATION}

\section{A. Equation error cost function}

The information embedded in the three microphone signals can be used to estimate the parameters via optimization, which requires the definition of an error and associated cost function. To this purpose, consider employing (13) with two different microphone pairs $\left(\mathcal{M}_{1}, \mathcal{M}_{0}\right)$ and $\left(\mathcal{M}_{1}, \mathcal{M}_{2}\right)$, resulting into two separate estimates of the forward traveling wave at the position of $\mathcal{M}_{1}$,

$$
\begin{aligned}
& \left.\hat{P}_{1}^{+}\right|_{10}=\frac{P_{1}-H_{1}^{(1+M)} P_{0}}{1-H_{1}^{2}}, \\
& \left.\hat{P}_{1}^{+}\right|_{12}=\frac{P_{1}-H_{1}^{(1+M)} H_{2}^{(1+M)} P_{2}}{1-H_{1}^{2} H_{2}^{2}} .
\end{aligned}
$$

At first sight, it may seem that simply squaring the estimator difference $\left.\hat{P}_{1}^{+}\right|_{10}-\left.\hat{P}_{1}^{+}\right|_{12}$ and summing over a range of frequencies would give a suitable cost function to be minimized. However, because of the system poles (the denominator roots), such a cost function is characterized by many local minima. A similar issue arises in adaptive filter theory in which evaluating the estimator difference would amount to a recursive filtering operation. A common way to address this problem is to break the recursion by replacing the feedback to the estimated filter with the reference signal, which leads to the so-called equation error method. ${ }^{30}$ For the problem at hand, an equation error can be obtained by multiplying the estimator difference with $\left(1-H_{1}^{2}\right)\left(1-H_{1}^{2} H_{2}^{2}\right) H_{1}^{-(1+M)}$, which yields

$$
\begin{aligned}
E^{\prime}= & H_{1}^{(1-M)}\left(1-H_{2}^{2}\right) P_{1}+\left(H_{1}^{2} H_{2}^{2}-1\right) P_{0} \\
& +H_{2}^{(1+M)}\left(1-H_{1}^{2}\right) P_{2},
\end{aligned}
$$

or, in terms of the microphone signals, after multiplying with $F_{1} F_{2}$, 


$$
\begin{aligned}
E= & H_{1}^{(1-M)}\left(1-H_{2}^{2}\right) F_{2} S_{1}+\left(H_{1}^{2} H_{2}^{2}-1\right) F_{1} F_{2} S_{0} \\
& +H_{2}^{(1+M)}\left(1-H_{1}^{2}\right) F_{1} S_{2} .
\end{aligned}
$$

For an idealized system, in which $F_{1}=F_{2}=M=0$ and $H_{1}=H_{2}$, Eq. (18) reduces to a preliminary formulation, ${ }^{15}$ which uses the more intuitive definition of the error as the difference between the pressure directly measured at the central microphone and an estimate of it calculated from $P_{1}$ and $P_{2}$.

A cost function that can be applied to a set of microphone signals of finite length $N$ sampled at a rate $f_{s}=1 / T$ can thus be obtained by evaluating the equation error spectrum at a selected set of frequencies of interest,

$$
\xi(\mathbf{v})=\sum_{k=k_{1}}^{k=k_{2}}|E[k]|^{2},
$$

where $E[k]$ denotes the $k$ th component of the equation error spectrum, corresponding to the frequency $f_{k}=k f_{s} / N$. Where needed, the immunity to noise can be improved by evaluating the power spectrum $|E|^{2}$ using Welch's method, ${ }^{31}$ which averages over a successive set of overlapping windowed data sub-segments, at the price of a reduced resolution in either frequency or time.

It is worthwhile noting that the bandwidth restriction on the wave separation due to singularities mentioned in Sec. II A does not apply to the parameter estimation procedure. That is, the fact that the term $|E[k]|^{2}$ drops toward zero at singular frequencies does not have a detrimental effect on the estimation, so a wider range of frequencies can be used. The estimation bandwidth is constrained, however, by the plane-wave assumption, which holds up to about the upperlimit frequency, ${ }^{11}$

$$
f_{\mathrm{u}}=\frac{1.84 c \sqrt{1-M^{2}}}{2 \pi a} .
$$

\section{B. Optimization}

The estimation problem can now be defined as finding the optimum vector $\stackrel{\circ}{\mathbf{v}}$ of $Q$ parameters that minimizes the cost function

$$
\stackrel{\circ}{\mathbf{v}}=\underset{\mathbf{v} \in \mathbb{R}^{Q}}{\operatorname{argmin}}\{\xi(\mathbf{v})\} .
$$

This is a nonlinear optimization problem that can be solved using standard iterative methods, provided that convergence to local minima can be avoided. A practical way to investigate the search space is through the use of acquisition data generated with simulations of the system in Fig. 2. For example, Fig. 3 shows $\xi$ as a function of $\tau_{1}$ and $\tau_{2}$, as evaluated via simulations for a white Gaussian noise source. For Fig. 3(a), the sum in (19) is limited between 0 and $8 \mathrm{kHz}$, while for Fig. 3(b), it is limited between 0 and $16 \mathrm{kHz}$. The parameter values used in the simulations are: $\tau_{1}=\tau_{2}=\tau_{0}$ $=0.05826, g=g_{0}=0.76 \mathrm{~Hz}^{\frac{1}{2}}, a_{1}=a_{2}=1, \quad \delta_{1}=\delta_{2}=0$, and $M=0$. The nominal values $\tau_{0}$ and $g_{0}$ were calculated
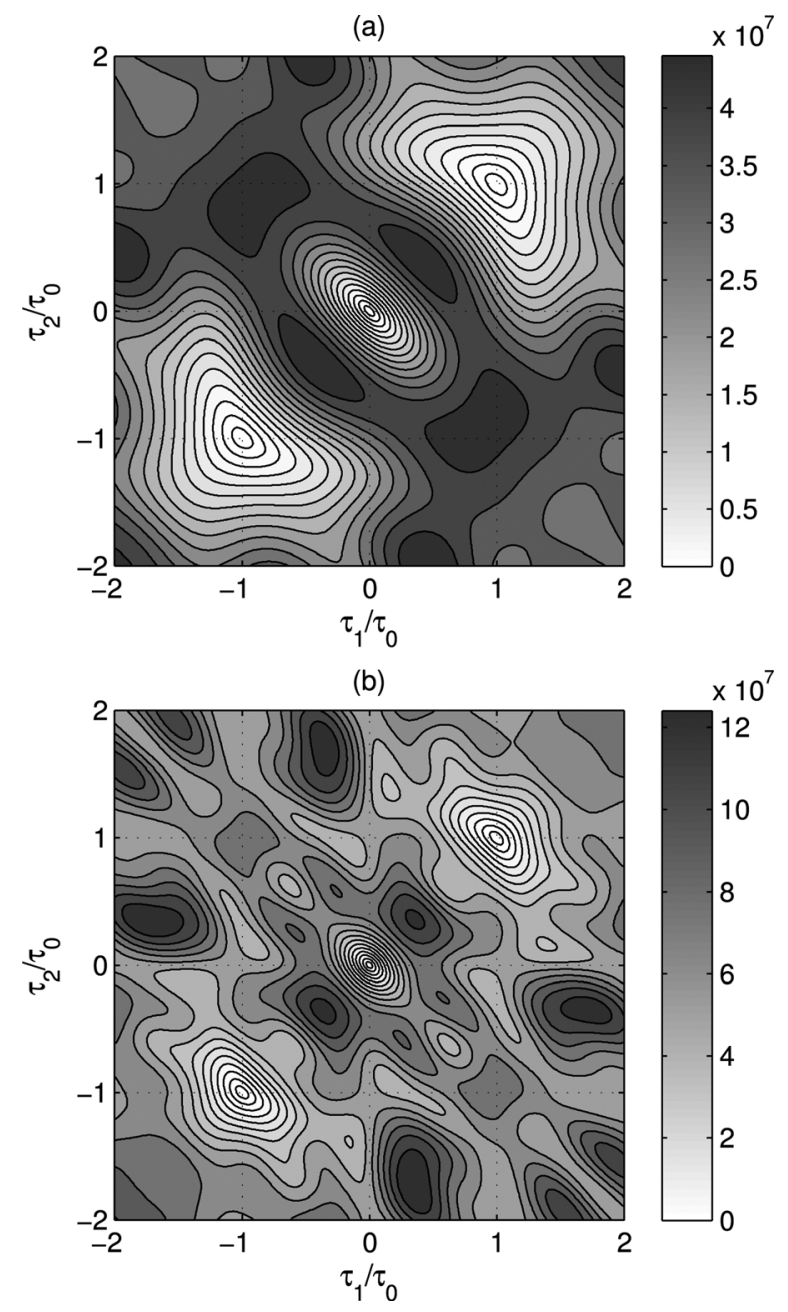

FIG. 3. Contour plots of $\xi$ as a function of $\tau_{1}$ and $\tau_{2}$ as evaluated for frequencies up to $8 \mathrm{kHz}$ (a) and $16 \mathrm{kHz}$ (b). The physically correct target minimum lies at coordinates $(1,1)$.

from air constants as predicted by theory ${ }^{26}$ for the room temperature recorded during the validation experiments described in Sec. IV and assuming the inter-microphone distances to equal the nominal value $d=2 \mathrm{~cm}$. In evaluating the cost function, all parameters apart from $\tau_{1}$ and $\tau_{2}$ were set at the nominal theory values used in the simulation.

As can be seen from the contour plots in Fig. 3, the cost function may contain several local minima. However, good initial guesses of most of the parameters are available; in particular, the values of $\tau_{1}, \tau_{2}$, and $g$ are approximately known from theory. This makes the optimization problem tractable with the use of local (unconstrained) minimization methods. ${ }^{32}$ The Nelder-Mead simplex method ${ }^{33}$ converges sufficiently fast in this case and is used for all results presented here. Alternatively, better convergence may be obtained using gradient-based methods. More specifically, because of the frequency-domain formulation, it is possible to obtain analytical expressions of the first and second order derivatives of the cost function ${ }^{24}$ and thus the Hessian matrix of $\xi$, which enables (21) to be solved using for example Newton's method. ${ }^{32}$

Comparing the two contour plots shows that the basins of convergence become smaller when a larger frequency 
range is used in (19). This means that the concavity of the basin around the optimum is more pronounced for a larger bandwidth, which, in the presence of extraneous measurement noise, leads to a smaller variance in the estimate. Hence if needed, it can be ensured that the initial guess falls into the correct basin by first estimating on a smaller frequency range, after which the estimate can be improved with a second estimation on a larger frequency range.

Further simulations, the results of which are omitted here for brevity, show that the cost function is similarly dependent on the channel amplitudes $\left(a_{1}, a_{2}\right)$ but in comparison is considerably less sensitive to changes in the parameters $g, \delta_{1}, \delta_{2}$, and $M$. These smaller dependencies imply that accurate measurements in $g$ or $M$ are possible only when all the parameters are estimated with high accuracy. This suggests that, for example, the measurement of $M$ can in itself be used as a way of assessing the validity and accuracy of the estimation procedure. Hence for experiments in which no direct separation reference measure is available, such as those discussed in Sec. V, wave separation may be studied and assessed indirectly through Mach number estimations.

\section{Noise influence}

Experimentally acquired microphone signals are inevitably contaminated with extraneous measurement noise. This will generally result in a variance in the estimated parameters and in addition an estimation bias may be observed. Such noise effects are easily reduced by averaging, but there is far less scope for this when tracking time-varying parameters. Therefore the dependence of the estimates for $\tau_{1}, g$, and $M$ on the signal-to-noise ratio (SNR) is investigated in this section by estimating these three tracking parameters concurrently from simulations in which independent white Gaussian noise is added to each of the microphone signals. The target parameters are $\tau_{0}, g_{0}$, and $M_{0}=0.01$, and the cost function evaluation is limited between 0 and $16 \mathrm{kHz}$, evaluated from a window of 8192 samples. In the estimation, the ratio $r=\tau_{2} / \tau_{1}=1$ is used, and all the other parameters are assumed constant and held fixed. For each SNR value, the simulation and subsequent estimation were carried out 1000 times, so that the mean value and the standard deviation can be obtained. Figure 4 shows the results for signal-to-noise power ratios between 15 and $40 \mathrm{~dB}$. The larger bias in the $g$ estimate reflects the fact that $\xi$ is relatively insensitive to changes in $g$ near the optimum. Figure 4(c) shows that the bias in $M$ is small compared to the variance.

\section{VALIDATION}

\section{A. Separation index}

To assess the proposed method of calibration via optimization, a measure is required of how well the optimized parameters describe the system behavior. In principle, this could be done by evaluating the accuracy and precision of the individual parameters, but it is difficult to obtain reliable reference measures of each of these. ${ }^{34}$ Moreover, a more meaningful and practical measure would express the

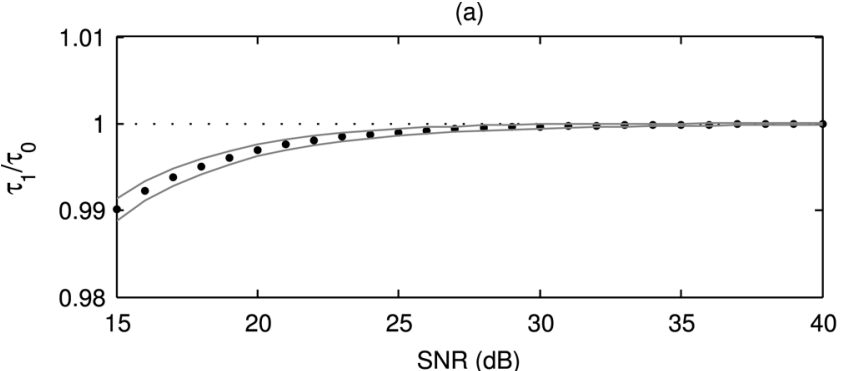

(b)

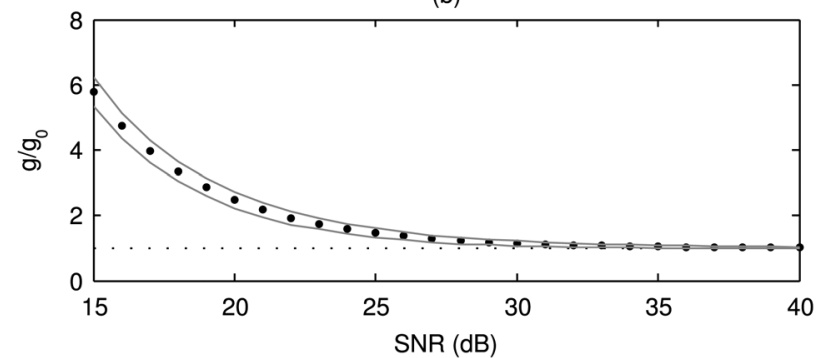

(c)

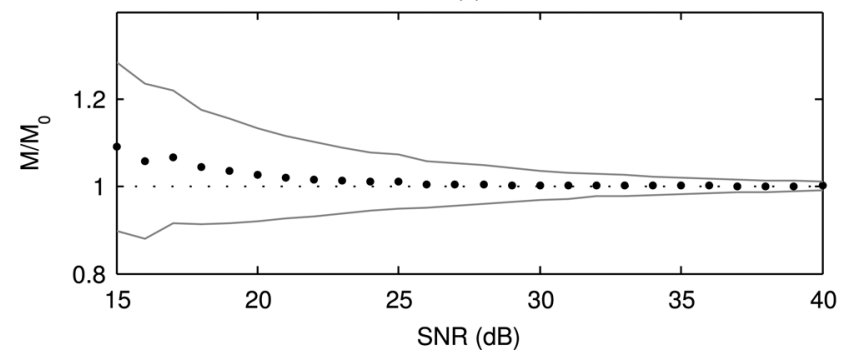

FIG. 4. Influence of measurement noise on the estimates of the tracking parameters $\tau_{1}, g$, and $M$ when using a white Gaussian noise source signal and an estimation window length of 8192 samples. Shown are the mean (dots) and the standard deviation (gray lines) over 1000 realizations.

accuracy in terms of a general target result, preferably with a single number so that comparisons between methods is straightforward. Given that all 1-D acoustic waveguide measurement techniques can be considered as wave separation methods, the proposed metric is therefore a number indicating how well the waves are separated, which in the following is referred to as the Separation Index. To formulate the index, the estimated waves need to be compared to reliable reference signals. A simple way to obtain these experimentally is by sending a source signal of short duration into the measuring tube, such that the initial forward and backward traveling waves do not overlap and can be temporally separated, as in pulse reflectometry. ${ }^{4,35}$ A filtered version of the first incident wave signal at $\mathcal{M}_{0}$ can then be obtained by simply "gating" the mid-point microphone signal. Provided that the end of the measuring tube is sufficiently reflective, a first reflected wave signal is obtained similarly (see, for example, the measured signal plotted in Fig. 5). The respective errors in estimating these wave signals may then be computed as

$$
\begin{aligned}
& \phi^{+}=\sum_{n=n_{f}}^{n_{f}+N-1}\left(s_{0}[n]-\hat{s}_{0}^{+}[n]\right)^{2}, \\
& \phi^{-}=\sum_{n=n_{b}}^{n_{b}+N-1}\left(s_{0}[n]-\hat{s}_{0}^{-}[n]\right)^{2}=\sum_{n=n_{b}}^{n_{b}+N-1}\left(\hat{s}_{0}^{+}[n]\right)^{2},
\end{aligned}
$$




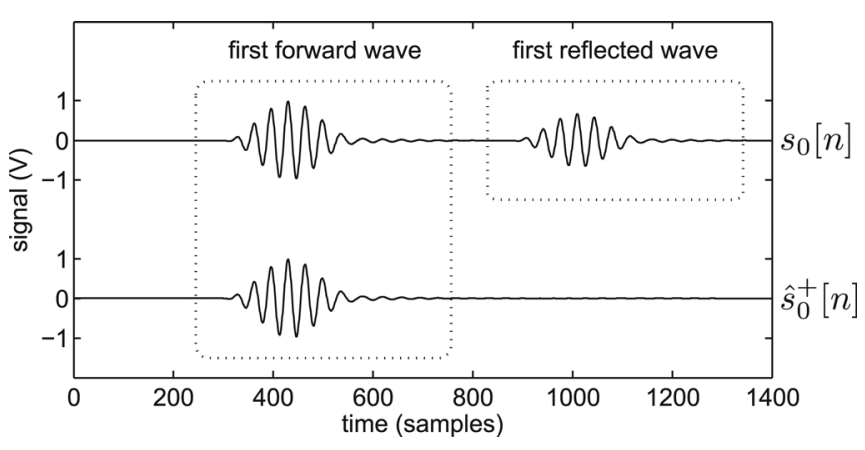

FIG. 5. Acquired signal at $\mathcal{M}_{0}$ after averaging over 100 acquisitions with a windowed $3 \mathrm{kHz}$ sine wave source signal. The forward wave estimate obtained with (28) is also shown.

where $\hat{s}_{0}^{+}[n]$ and $\hat{s}_{0}^{-}[n]$, respectively, are the estimated forward and backward waves, both filtered by the channel response $G_{0}$. The time windows $\left[n_{f}, n_{f}+N-1\right]$ and $\left[n_{b}, n_{b}+N-1\right]$ are chosen such that the compact forward and backward wave signals are cleanly captured from the mid-point microphone signal. However, neither $\phi^{+}$nor $\phi^{-}$ can by itself serve as an adequate measure because a null error would result whenever $\hat{s}_{0}^{ \pm} \equiv s_{0}$, a situation that can occur for some choices of the parameters not corresponding to separating the waves. In addition, both error terms are proportional to the amplitude of the acquired signals. It follows that what should be measured is the sum of the normalized errors in the reconstructions of the incident and reflected waves,

$$
\phi=\frac{\phi^{+}}{\eta^{+}}+\frac{\phi^{-}}{\eta^{-}}
$$

where

$$
\eta^{+}=\sum_{n=n_{f}}^{n_{f}+N-1} s_{0}^{2}[n], \quad \eta^{-}=\sum_{n=n_{b}}^{n_{b}+N-1} s_{0}^{2}[n]
$$

are the respective normalizing energies. However, depending on the spectral content of the source signal, this measure may not be significant at all frequencies. The performance of a separation algorithm is therefore generally best studied within a specified bandwidth, for which (24) is re-written in a frequency-dependent form

$$
\Phi[k]=\frac{\left|S_{0, \mathrm{f}}[k]-\hat{S}_{0, \mathrm{f}}^{+}[k]\right|^{2}}{N \eta^{+}}+\frac{\left|\hat{S}_{0, \mathrm{~b}}^{+}[k]\right|^{2}}{N \eta^{-}},
$$

where $S_{0, \mathrm{f}}$ and $\hat{S}_{0, \mathrm{f}}^{+}$are the DFTs of, respectively, $s_{0}[n]$ and $\hat{s}_{0}^{+}[n]$ for $n_{f} \leq n<n_{f}+N$, and $\hat{S}_{0, \mathrm{~b}}^{+}$is the DFT of $\hat{s}_{0}^{+}[n]$ for $n_{b} \leq n<n_{b}+N$. The separation index for a specified bandwidth ranging between frequencies indexed by $k_{1}$ and $k_{2}$ can then be defined in decibels as

$$
\Psi=10 \log _{10}\left(\sum_{k=k_{1}}^{k_{2}} \Phi[k]\right)
$$

Note that for the calculation of $\Psi$, only the forward wave estimate $\hat{S}_{0}^{+}$is required; this can be calculated from (13) for the microphone pair $\left(\mathcal{M}_{0}, \mathcal{M}_{2}\right)$ with $M=0$ as

$$
\hat{S}_{0}^{+}=G_{0} \hat{P}_{0}^{+}=\frac{S_{0}-\left(\stackrel{\circ}{H}_{2} / \stackrel{\circ}{F}_{2}\right) S_{2}}{1-\dot{\circ}_{2}^{2}},
$$

where $\stackrel{\circ}{H}_{2}$ is the transfer function $\mathrm{H}_{2}$ evaluated with the optimum parameter vector $\dot{\mathbf{v}}$ and $\stackrel{\circ}{F}_{2}$ is the corresponding interchannel complex amplitude of $\mathcal{M}_{2}$.

\section{B. Measurement apparatus and procedure}

The measurement apparatus used in the validation experiments is shown schematically in Fig. 6. Endevco piezo-resistive microphones (model $85070 \mathrm{C}-1$ ) and a JBL compression driver (model 2426J) are used to, respectively, sense and drive the acoustic field, while a National Instruments USB-6251 acquisition board is employed for digital-to-analog conversion to 16 -bit signals at a $100 \mathrm{kHz}$ sample rate. A metal cap is placed at the end of the measuring tube to maximize reflections.

Two acquisitions are carried out, one for pre-calibration and one for testing wave separation for a specific test signal. In the pre-calibration acquisition, the maximum-length sequence (MLS) method ${ }^{36}$ is used to measure the impulse responses at the microphone positions. A 19th-order MLS is sent to the compression driver and the signals from the three microphones acquired. Next, the cross-correlation between the MLS input signal and each of the three acquired microphone signals is computed; this yields three impulse responses, the spectra of which are used as $S_{1}, S_{0}$, and $S_{2}$ in the algorithm for estimating the system parameters as explained in Sec. III. In the second acquisition, a test signal of short duration is fed to the compression driver, for example a Hanning-windowed sine wave. Averaging the microphone signals over multiple acquisitions can be used to improve the SNR. Figure 5 shows the first 1400 samples of the signal obtained by averaging over 100 acquisitions at $\mathcal{M}_{0}$ for a short windowed sine wave source signal. For comparison, the estimated forward wave signal calculated with (28) after calibration via optimization is also plotted; this demonstrates the cancellation of the backward-traveling wave. As explained in Sec. IV A, the actual forward traveling wave signal can in this case be extracted from the acquired microphone signal by gating.

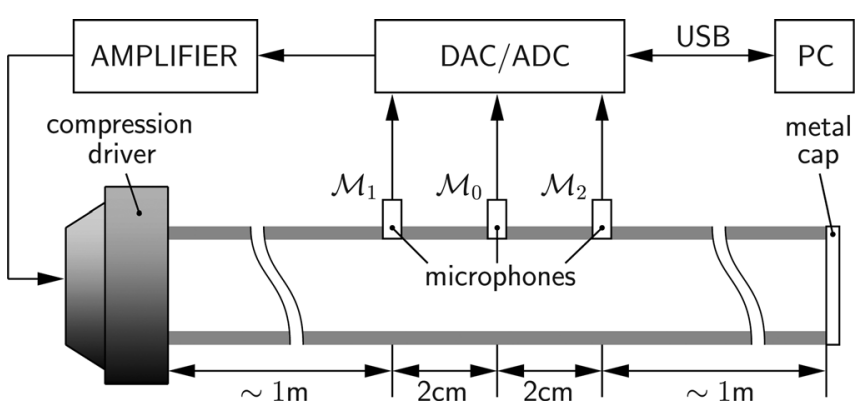

FIG. 6. Measurement apparatus used in the validation experiments. The internal diameter of the cylindrical measuring tube is $12.7 \mathrm{~mm}$, and the microphone diameter is $2.5 \mathrm{~mm}$. 


\section{Reference experiment}

For constant propagation conditions, the measurement system can also be calibrated through pre-measured responses. The estimated forward wave thus obtained can be used as a reference, by separation index comparison. Referring again to Fig. 2, the signals $s_{0}$ and $s_{2}$ acquired by microphones $\mathcal{M}_{0}$ and $\mathcal{M}_{2}$ can be written as a function of the forward pressure wave at $\mathcal{M}_{0}$. Again taking $M=0$, the frequency-domain relationships are

$$
\begin{aligned}
& S_{0}=G_{0}\left(1+H_{2}^{2} R\right) P_{0}^{+}, \\
& S_{2}=G_{2}\left(H_{2}+H_{2} R\right) P_{0}^{+} .
\end{aligned}
$$

By writing the forward and the backward traveling wave components separately,

$$
\begin{aligned}
& S_{0, \mathrm{f}}=G_{0} P_{0}^{+}, \quad S_{0, \mathrm{~b}}=G_{0} H_{2}^{2} R P_{0}^{+}, \\
& S_{2, \mathrm{f}}=G_{2} H_{2} P_{0}^{+}, \quad S_{2, \mathrm{~b}}=G_{2} H_{2} R P_{0}^{+},
\end{aligned}
$$

the following transfer functions can be defined:

$$
\frac{S_{2, \mathrm{f}}}{S_{0, \mathrm{f}}}=\frac{G_{2}}{G_{0}} H_{2}=H_{2}^{\prime}, \quad \frac{S_{0, \mathrm{~b}}}{S_{2, \mathrm{~b}}}=\frac{G_{0}}{G_{2}} H_{2}=H_{2}^{\prime \prime} .
$$

This implies that $H_{2}=\sqrt{H_{2}^{\prime} H_{2}^{\prime \prime}}$, and that, analogous to (28), an estimate of the (filtered) forward wave is

$$
\hat{S}_{0}^{+}=\frac{S_{0}-H_{2}^{\prime \prime} S_{2}}{1-H_{2}^{\prime} H_{2}^{\prime \prime}} .
$$

Measures of $H_{2}^{\prime}$ and $H_{2}^{\prime \prime}$ can be evaluated from (33) after extracting the relevant forward and backward waves from the same pre-calibration acquisition as used for the optimization. That is, $S_{0, \mathrm{f}}$ and $S_{2, \mathrm{f}}$ are obtained by isolating the first forward wave from each of the respective impulse responses measured using the MLS method as explained in Sec. IV B and taking the DFTs after zero-padding from 512 to 8192 samples. The transfer function $H_{2}^{\prime}$ is then calculated by dividing these spectra. The second transfer function $H_{2}^{\prime \prime}$ is determined in the same fashion, where $S_{2, \mathrm{~b}}$ and $S_{0, \mathrm{~b}}$ are the DFTs of the signals obtained by isolating the first endreflection in each of the two measured impulse responses.

\section{Results}

The proposed method of calibration via optimization is compared in this section to results obtained with the reference experiment. To get further insight into the effect of the calibration on $\Psi$, a third estimate is calculated in which the transfer function is evaluated from theory, thus applying (28) but with $\stackrel{\circ}{F}_{2}$ and $\stackrel{\circ}{H}_{2}$, respectively, replaced by 1 and $H_{0}$, the latter being the transfer function evaluated using the nominal theory parameters as used for the simulated cost function results in Sec. III B.

Table I lists the separation index results for two different test signals, a white Gaussian noise and a $3 \mathrm{kHz}$ sine wave, both windowed with a 256-sample length Hanning window. For the white Gaussian noise, the sum in (27) is evaluated for frequencies between 0.1 and $8 \mathrm{kHz}$, while for the $3 \mathrm{kHz}$ sine wave, the used limits are 2 and $4 \mathrm{kHz}$. The results are obtained from a single acquisition as well as by averaging over 16 and 100 acquisitions. Figure 7 shows the separation index as a function of frequency. As can be expected, the results significantly improve with averaging. More importantly, they demonstrate that (1) calibration generally improves the estimation in comparison to using theoretical parameter values and (2) the optimization method gives results similar to those obtained with the reference experiment. Note that the results obtained with the theoretical parameters depend on having conditions that allow prediction from an available temperature measurement; this is generally not the case in the envisaged scenarios in which any reliance on theory will thus cause larger wave separation errors.

\section{MEASUREMENT WITH MEAN FLOW}

In this section, the tracking performance of the proposed estimation method is investigated by estimating the parameters from microphone acquisitions obtained during a period of time-varying mean flow through two separate experiments.

\section{A. Dry air with CPU fan flow}

For this experiment, the measuring tube is terminated on one end by a compression driver fed with a white noise broadband signal. The other end is fitted with a funnel and a small fan taken from a CPU cooling system (see Fig. 8). An airhole is placed just before the driver to allow a steady circulation of the air. This arrangement allows generating a controlled flow while avoiding as much as possible any further perturbations in the conditions.

\begin{tabular}{|c|c|c|c|c|c|c|c|c|}
\hline & & \multirow[b]{2}{*}{ Averaging: } & \multicolumn{3}{|c|}{ White Gaussian noise } & \multicolumn{3}{|c|}{$3 \mathrm{kHz}$ sine wave } \\
\hline & & & 1 & 16 & 100 & 1 & 16 & 100 \\
\hline Theory & $\hat{S}_{0}^{+}=\frac{S_{0}-H_{0} S_{2}}{1-H_{0}^{2}}$ & & -19.2 & -25.2 & -25.2 & -31.8 & -31.9 & -32.0 \\
\hline Reference & $\hat{S}_{0}^{+}=\frac{S_{0}-H_{2}^{\prime \prime} S_{2}}{1-H_{2}^{\prime} H_{2}^{\prime \prime}}$ & & -20.7 & -34.2 & -37.9 & -43.2 & -49.4 & -50.0 \\
\hline Optimization & $\hat{S}_{0}^{+}=\frac{S_{0}-\left(\stackrel{\circ}{H}_{2} / \stackrel{\circ}{F}_{2}\right) S_{2}}{1-\stackrel{\circ}{H}_{2}^{2}}$ & & -21.7 & -34.7 & -39.1 & -46.3 & -48.3 & -49.8 \\
\hline
\end{tabular}

The experiment consists in the acquisition of two sequences; the first, with the fan switched off, is used to precalibrate the system, estimating "zero-flow values" of the first seven parameters of the vector in (14). In the second

TABLE I. Separation index results (in $\mathrm{dB}$ ). 
(a)

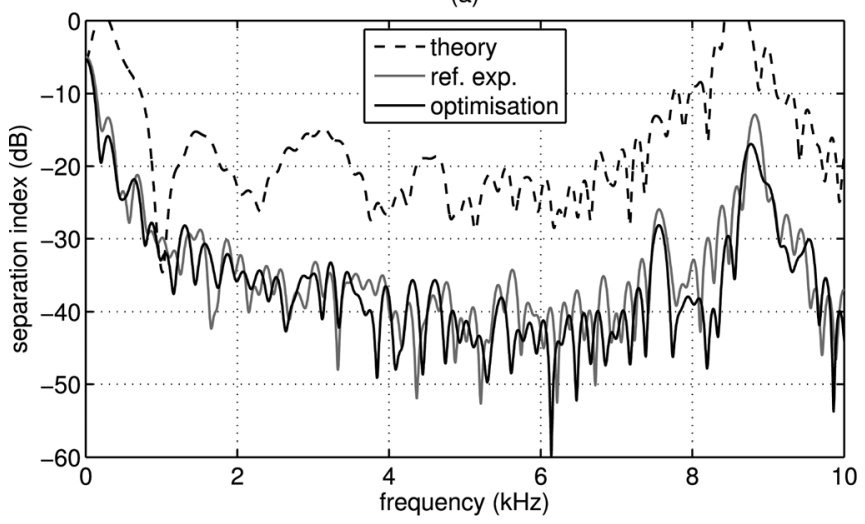

(b)

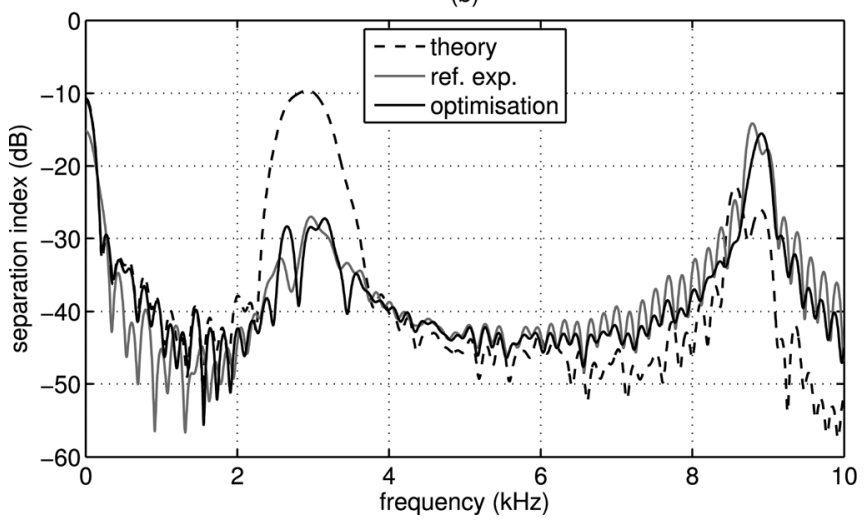

FIG. 7. Separation index as a function of frequency from 100 averaged acquisitions for a windowed white Gaussian noise source signal (a) and windowed $3 \mathrm{kHz}$ sine wave (b). Singular frequencies are positioned at 0 and $8.58 \mathrm{kHz}$.

experiment, the fan is first switched on, left to run for a few seconds, and then switched off. The expected result is thus a non-negative flow velocity that builds up from zero to a steady level and then decays back to zero during the freerotating period in which the fan loses its kinetic energy.

Because the parameters $\delta_{1}$ and $\delta_{2}$ directly affect the flow velocity estimate, they must be estimated from the precalibration acquisition and kept fixed in the second acquisition. Block-wise estimation from the second acquisition is then performed in two different ways, first by estimating all the remaining six parameters, and second by keeping $r, a_{1}$, and $a_{2}$ fixed, thus estimating only the waveguide parameters $\tau_{1}, g$, and $M$. This allows verifying to what extent the assumption that only the latter vary with the conditions holds. The resulting evolutions in $\tau_{1}, g$, and $M$ are shown in Fig. 9 and indicate that not only $M$ but also $\tau_{1}$ and $\tau_{2}$ are time-varying in this case, while $g$ mainly shows a statistical

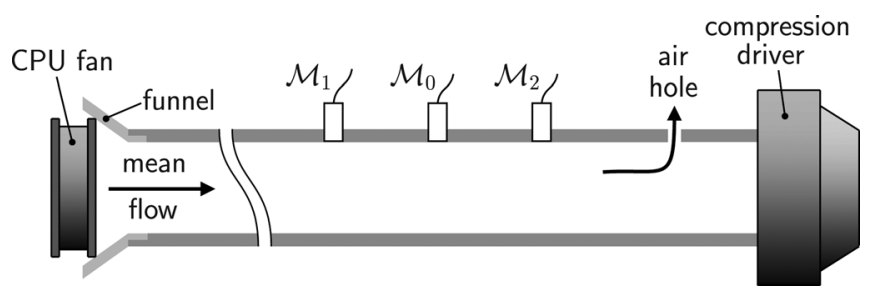

FIG. 8. Experimental setup used for measurements with mean flow. The internal diameter of the cylindrical measuring tube is $15 \mathrm{~mm}$. (a)

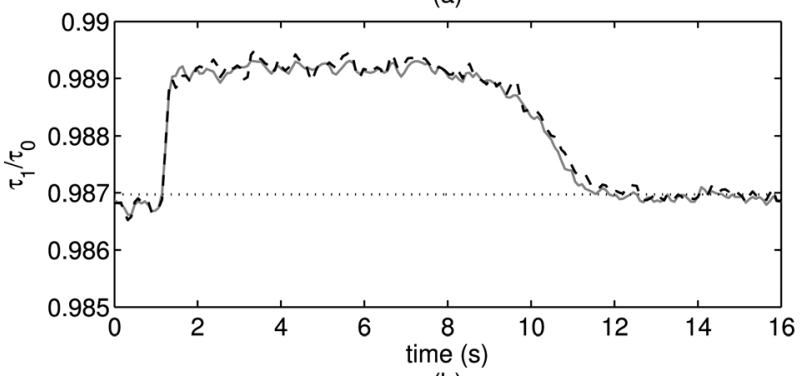

(b)

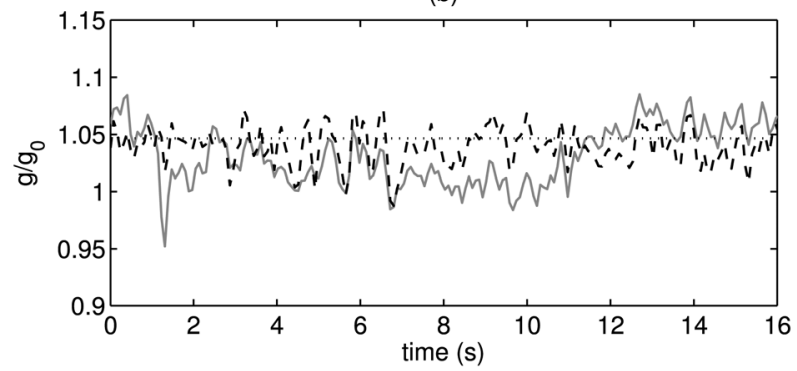

(c)

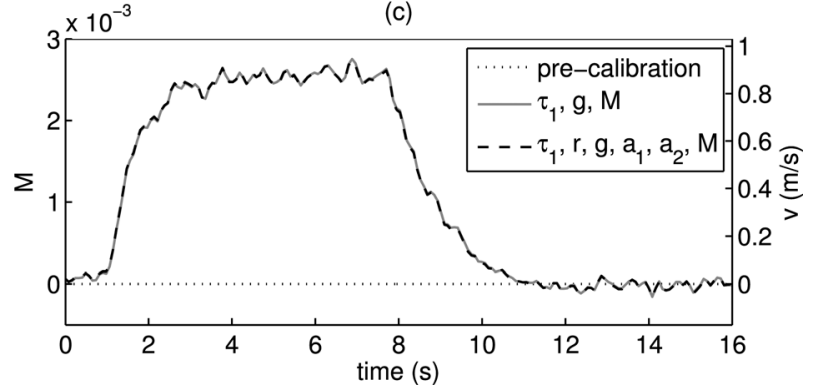

FIG. 9. Evolution of $\tau_{1}, g$, and $M$, as estimated under dry conditions with a fan generating a mean flow. The estimates were computed every 8192 samples, using a 16384-sample analysis window. The legend in (c) indicates which parameters were estimated.

variation around its mean; the latter trend was also observed in the other parameters estimated $\left(r, a_{1}, a_{2}\right)$, although with considerably smaller variance. Figure 9 (c) confirms for this case that it is reasonable to assume that all system parameters apart from $\tau_{1}, \tau_{2}$, and $M$ are constant. Furthermore, the mean flow velocity $(v=M c)$ follows the expected trend: The null flow is correctly estimated, a steady state period can be observed, and even the first-order response of the fan motor to the voltage step is visible. The small step in $\tau_{1}$ at $t=1.2 \mathrm{~s}$ suggests a change in the wave speed corresponding to a temperature drop of about $1.1^{\circ} \mathrm{C}$, a possible explanation of which is that the introduction of a mean flow may cause a change in the heat transfer between the air and the tube wall.

\section{B. Flow from breath}

Given that the flow velocity estimation in a controlled environment returns plausible results, the experiment is repeated but with the funnel and fan replaced with a (reedless) clarinet mouthpiece and supplying an airflow by a human blowing moist air into the measuring tube; this provides conditions similar to those in a wind instrument. By exclusively exhaling during the acquisition, the mean flow velocity is again expected to be non-negative. In addition, an effort was made to generate a monotonic rise and fall in mean flow. Figure 10 shows the resulting parameter 
(a)

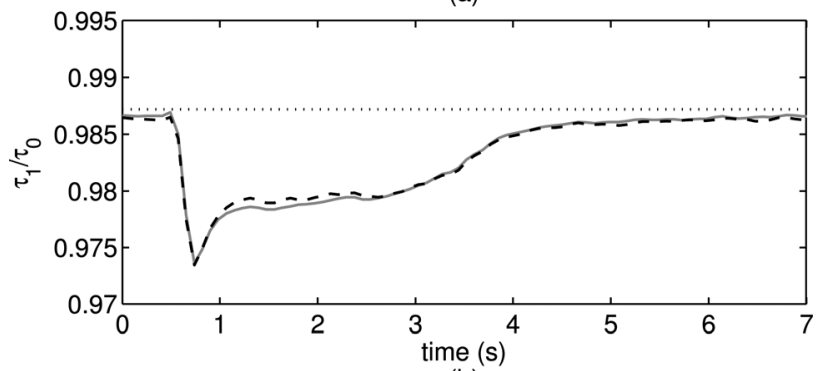

(b)

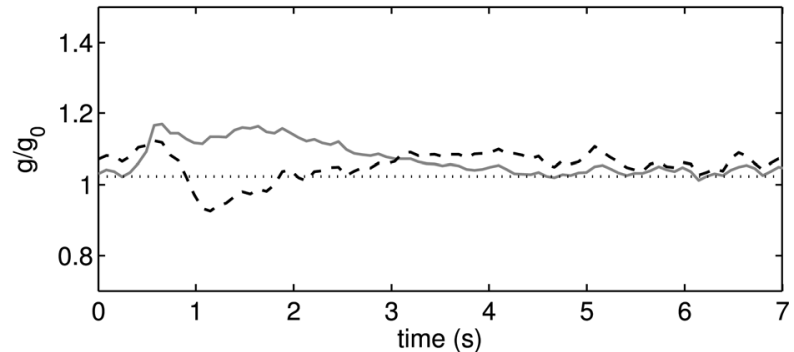

(c)

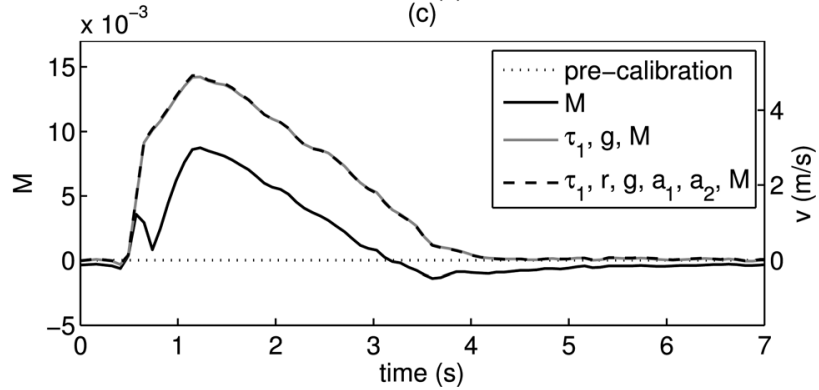

FIG. 10. Evolution of $\tau_{1}, g$, and $M$, as estimated under fluctuations in temperature and humidity effected by blowing into the measuring tube. The estimates were computed every 8192 samples, using a 16384-sample analysis window. The legend in (c) indicates which parameters were estimated.

evolutions, obtained similarly to the experiment with the dry air flow. The evolution of $M$ is again insensitive to whether the parameters $r, a_{1}$, and $a_{2}$ are estimated or held fixed. A rigorous analysis of the causes behind the visible fluctuations in $\tau_{1}$ and $g$ is beyond the scope of this study; in particular the fluctuations in $g$ may be due to many different factors and show no consistency between the two estimations. One main trend that proved consistent across all measurements of this type can be explained though. That is, the significant drop in $\tau_{1}$ at the point where the mean flow starts to rise marks the arrival of air of higher temperature and humidity, causing a sudden increase in wave speed.

Figure 10(c) also shows an additional estimation from the same data in which all parameters except $M$ are held fixed. This results into an erratic, non-smooth flow evolution, and even results into a period of negative estimated flow, which is not consistent with the applied input. This result exemplifies the notion that under transient propagation conditions, the system calibration must be adaptive in its timevariant parameters.

\section{CONCLUDING REMARKS}

The problem of calibrating two-microphone wave separation methods under time-varying conditions can be made tractable by physical parameterization of the intermicrophone propagation transfer function. It has been shown that a three-microphone measurement configuration can be used to calibrate and track the relevant parameters. For measurements under constant conditions, the proposed method of calibration via optimization has been shown in Sec. IV to perform very similarly to a more standard calibration method based on pre-measuring system responses. This result validates the basic methodology; in particular, it justifies the way in which the three-microphone measurement system has been parameterized in Sec. IIC. The measurements with mean flow in Sec. $\mathrm{V}$ demonstrate that adaptive calibration is not only possible but indeed necessary when several condition aspects, such as temperature and humidity, are variable. Although the estimated Mach numbers are not directly compared to a reference measure, the observed mean flow evolutions are plausible and consistent with the applied inputs.

These results pave the way for addressing a range of challenging measurement problems. Of particular interest to the authors is the determination of the acoustic variables inside a wind instrument under playing conditions in a nonintrusive manner in the sense that there is minimal interference with the normal functioning and musician's control of the instrument. The proposed methodology has in fact already been successfully employed as such, in the form of wave separation in a simplified clarinet for the purpose of estimating the reed parameters by inverse modeling. ${ }^{17}$ Although wave propagation in an instrument bore may become non-linear at high playing levels, ${ }^{37}$ this would not invalidate the basic two-microphone wave separation method because such effects are small over short distances, thus allowing for a locally linear approximation. ${ }^{21}$

The approach taken in the present study may also lend itself to improvements and extensions in other applications. For example, a more accurate impedance measurement in the presence of a mean flow may be possible if the Mach number is included in the calibration procedure. ${ }^{10}$ Generally, not requiring any additional measurements with special made-toprecision objects may represent a significant advantage in certain measurement scenarios. Finally, the ability of calibrating in-system on short signals could also be useful in control applications given that the existing control methods usually achieve the required efficiency and noise immunity at the cost of reducing the wave separation accuracy.

\section{ACKNOWLEDGMENTS}

The research was supported by the Engineering and Physical Sciences Research Council (UK), Grant No EP/D074983/1. The authors are grateful to David Sharp for his helpful comments on earlier versions of this text.

${ }^{1}$ R. Causse, J. Kergomard, and X. Lurton, "Input impedance of brass musical instruments-comparison between experiment and numerical models," J. Acoust. Soc. Am. 75, 241-254 (1984).

${ }^{2}$ V. Gibiat and F. Laloe, "Acoustical impedance measurements by the twomicrophone-three-calibration (TMTC) method," J. Acoust. Soc. Am. 88, 2533-2545 (1990).

${ }^{3} \mathrm{~N}$. Amir, U. Shimony, and G. Rosenhouse, "A discrete model for tubular acoustic systems with varying cross-section the direct and inverse problems. Part 2: Experiments," Acta Acust. Acust. 81, 463-474 (1995). 
${ }^{4}$ D. B. Sharp, "Acoustic pulse reflectometry for the measurement of musical wind instruments," Ph.D. thesis, University of Edinburgh, 1996, pp. 1-192.

${ }^{5}$ J.-P. Dalmont, C. J. Nederveen, V. Dubos, S. Ollivier, V. Meserette, and E. te Sligte, "Experimental determination of the equivalent circuit of an open side hole: Linear and non linear behaviour," Acta Acust. Acust. 88, 567-575 (2002).

${ }^{6}$ S. Rodriguez, V. Gibiat, A. Lefebvre, and S. Guilain, "The threemeasurement two-calibration method for measuring the transfer matrix," J. Acoust. Soc. Am. 129, 3056-3067 (2011).

${ }^{7}$ A. F. Seybert and D. F. Ross, "Experimental determination of acoustic properties using a two-microphone random-excitation technique," J. Acoust. Soc. Am. 61, 1362-1370 (1977).

${ }^{8} \mathrm{M}$. Abom and H. Boden, "Error analysis of two-microphone measurements in ducts with flow," J. Acoust. Soc. Am. 83, 2429-2438 (1988).

${ }^{9}$ S.-H. Jang and J.-G. Ih, "On the multiple microphone method for measuring in-duct acoustic properties in the presence of mean flow," J. Acoust. Soc. Am. 103, 1520-1526 (1998).

${ }^{10}$ S. Rodriguez, V. Gibiat, A. Lefebvre, and S. Guilain, "Input impedance in flow ducts: Theory and measurement," J. Acoust. Soc. Am. 132, 1494-1501 (2012)

${ }^{11}$ Y.-B. Kim and Y.-H. Kim, "A measurement method of the flow rate in a pipe using a microphone array," J. Acoust. Soc. Am. 112, 856-865 (2002).

${ }^{12} \mathrm{P}$. Dickens, J. Smith, and J. Wolfe, "Improved precision in measurements of acoustic impedance spectra using resonance-free calibration loads and controlled error distribution," J. Acoust. Soc. Am. 121, 1471-1481 (2007).

${ }^{13}$ A. H. Benade and M. I. Ibisi, "Survey of impedance methods and a new piezo-disk-driven impedance head for air columns," J. Acoust. Soc. Am. 81, 1152-1167 (1987).

${ }^{14}$ J.-P. Dalmont, "Acoustic impedance measurement. Part I: A review," J. Sound Vib. 243, 427-439 (2001)

${ }^{15}$ M. van Walstijn and G. de Sanctis, "Towards physics-based re-synthesis of woodwind tones," in International Congress on Acoustics (ICA 2007), Madrid, Spain (2007).

${ }^{16}$ T. Smyth and J. S. Abel, "Toward an estimation of the clarinet reed pulse from instrument performance,” J. Acoust. Soc. Am. 131, 4799-4810 (2012).

${ }^{17} \mathrm{~V}$. Chatziioannou and M. van Walstijn, "Estimation of clarinet reed parameters by inverse modelling," Acta Acust. Acust. 98, 629-639 (2012).

${ }^{18}$ J. Guérard and X. Boutillon, "Real time acoustic travelling waves separation in a tube," in Proceedings of the 1997 International Symposium on Musical Acoustics, Edinburgh (1997).

${ }^{19}$ J. Guérard, "Numerical modeling and experimental simulation of acoustic systems: Application to musical instruments" (in French), Ph.D. thesis, Université de Paris VI, Paris, France, 1998, Chap. 4.

${ }^{20} \mathrm{P}$. Nauclér and T. Söderström, "Separation of waves governed by the onedimensional wave equation: A stochastic systems approach," Mech. Syst. Sig. Proc. 23, 823-844 (2009).
${ }^{21}$ P. L. Rendón, F. Orduña-Bustamante, D. Narezo, A. Pérez-López, and J. Sorrentini, "Nonlinear progressive waves in a slide trombone resonator," J. Acoust. Soc. Am. 127, 1096-1103 (2010).

${ }^{22}$ J. A. Kemp, M. van Walstijn, D. M. Campbell, J. P. Chick, and R. A. Smith, "Time domain wave separation using multiple microphones," J. Acoust. Soc. Am. 128, 195-205 (2010).

${ }^{23}$ J. A. Kemp, S. Bilbao, J. McMaster, and R. A. Smith, "Wave separation in the trumpet under playing conditions and comparison with time domain finite difference simulation," J. Acoust. Soc. Am. 134, 1395-1406 (2013).

${ }^{24} \mathrm{G}$. de Sanctis and M. van Walstijn, "A frequency domain algorithm for wave separation," in 12th International Conference on Digital Audio Effects (DAFx-09), Como, Italy (2009), pp. 498-505.

${ }^{25}$ A. D. Pierce, Acoustics: An Introduction to Its Physical Principles and Applications (AIP, New York, 1989), Chap. 10.

${ }^{26}$ D. H. Keefe, "Acoustical wave propagation in cylindrical ducts: Transmission line parameter approximations for isothermal and nonisothermal boundary conditions," J. Acoust. Soc. Am. 75, 58-62 (1984).

${ }^{27}$ M. van Walstijn, D. Campbell, J. Kemp, and D. Sharp, "Wideband measurement of the acoustic impedance of tubular objects," Acta Acust. Acust. 91, 590-604 (2005).

${ }^{28}$ T. Hélie and D. Matignon, "Diffusive representations for the analysis and simulation of flared acoustic pipes with visco-thermal losses," Math. Models Methods Appl. Sci. 16, 503-536 (2006).

${ }^{29}$ G. De Sanctis, "In-system parametric calibration for two-microphone wave separation in acoustic waveguides," Ph.D. thesis, Queen's University Belfast, Belfast, U.K., 2012, Chap. 2.

${ }^{30}$ G. C. Goodwin and K. S. Sin, Adaptive Filtering Prediction and Control (Prentice-Hall, Englewood Cliffs, NJ, 1984), Chap. 3.

${ }^{31} \mathrm{P}$. Welch, "The use of fast Fourier transform for the estimation of power spectra: A method based on time averaging over short, modified periodograms," IEEE Trans. Audio Electroacoust. 15, 70-73 (1967).

${ }^{32}$ A. R. Conn, N. I. M. Gould, and P. L. Toint, Trust-Region Methods (Society for Industrial and Applied Mathematics, Philadelphia, PA, 2000), Chap. 6.

${ }^{33}$ J. A. Nelder and R. Mead, "A simplex method for function minimization," Comput. J. 7, 308-313 (1965).

${ }^{34}$ G. de Sanctis and M. van Walstijn, "Calibration of apparatus for wave separation in wind instruments," J. Acoust. Soc. Am. 123, 3016 (2008).

${ }^{35}$ L. Sun and H. Hou, "Transmission loss measurement of acoustic material using time-domain pulse-separation method (L)," J. Acoust. Soc. Am. 129, 1681-1684 (2011).

${ }^{36}$ J. B. Borish and J. Angell, "An efficient algorithm for measuring the impulse response using pseudorandom noise," J. Audio Eng. Soc. 31, 478-488 (1983).

${ }^{37}$ A. Hirschberg, J. Gilbert, R. Msallam, and A. P. J. Wijnands, "Shock waves in trombones," J. Acoust. Soc. Am. 99, 1754-1758 (1996). 